\title{
2-Diphenylphosphinoyl-acetyl as a remote directing group for the highly stereoselective synthesis of $\beta$-glycosides
}

Xianglai Liu, ${ }^{1}$ Yetong Lin, ${ }^{1}$ Qianhui Sun, ${ }^{1}$ Huiyong Sun, ${ }^{1}$ Peng Xu,,${ }^{2}$ Guolong Li, ${ }^{1}$ Yingying Song,,${ }^{1}$ Weijia Xie, ${ }^{1}$ Haopeng Sun, ${ }^{1}$ Biao $\mathrm{Yu},{ }^{2}$ Wei $\mathrm{Li}^{1 *}$

${ }^{1}$ Department of Medicinal Chemistry, School of Pharmacy, China Pharmaceutical University, 639 Longmian Avenue, Nanjing, Jiangsu 211198, China

${ }^{2}$ State Key Laboratory of Bioorganic and Natural Products Chemistry, Shanghai Institute of Organic Chemistry, Chinese Academy of Sciences, 345 Lingling Road, Shanghai 200032, China

E-mail: wli@cpu.edu.cn

\begin{abstract}
The configuration of the anomeric glycosidic linkages is crucial for maintaining the biological functions and activities of carbohydrate molecules. However, their stereochemistry control in glycosylation represents one of the most challenging tasks in carbohydrate chemistry. In this report, the easily accessible 2-diphenylphosphinoyl-acetyl (DPPA) group was developed as a highly stereodirecting group for catalytic glycosylation via hydrogen-bond mediated delivery of the alcoholic acceptors. TMSOTf-catalyzed glycosylation with 6-O-DPPA glycosyl imidate donors displayed excellent $\beta$-selectivity and broad substrate scope, particularly applicable to synthesize the challenging $\beta$-configured 2-deoxy-and 2-azido-2-deoxy-glycosides from electron-deficient or bulky acceptors. Chemoselective removal of the DPPA group could be readily achieved under the mild catalysis of $\mathrm{Ni}(\mathrm{OTf})_{2}$, and further application was demonstrated in the synthesis of biologically important oligosaccharides, uronic acids, and 2,6-dideoxy-glycosides.
\end{abstract}


Carbohydrates are one of the essential biomolecules in life processes, and are persistently engaged in the occurrence and treatment of many diseases. ${ }^{1,2}$ The conformation and bioactivities of carbohydrates are significantly affected by the configuration of the anomeric glycosidic linkages in either $\alpha$ (usually axial for pyranosides) or $\beta$ (usually equatorial for pyranosides) orientation. However, a typic chemical glycosylation is promoted by acid and proceeds via $\mathrm{S}_{\mathrm{N}} 1$ pathway, giving a mixture of $\alpha$ - and $\beta$-glycosides (Fig. 1a). ${ }^{3,4}$ Hence, the stereochemistry control in glycosylation is one of the central topics and difficult tasks in carbohydrate chemistry, ${ }^{5-7}$ particularly in the synthesis of $\beta$-configured 2-deoxy-and 2-azido-2-deoxy-glycosides, ${ }^{8-14}$ which are important structural components or synthetic precursors of numerous drugs and bioactive molecules such as digoxin, landomycin, hyaluronic acid, and lipid A. ${ }^{15,16}$ Owing to the anomeric effect, glycosylation with 2-deoxy- or 2-azido-2-deoxy-glycosyl donors tends to give $\alpha$-glycosides as the major product. $3,4,10$ Thus, extensive studies have been devoted into the construction of the more challenging $\beta$-glycosides, ${ }^{8-14}$ employing $\alpha-$ donors/intermediates via $S_{N} 2 / S_{N} 2$-like pathway, ${ }^{17-20} \alpha$-face steric hindrance, ${ }^{21-24} 2$-halo participation, ${ }^{25,26} \beta$-glycosyl lithium, ${ }^{27,28}$ triflate acceptor, ${ }^{29}$ 2,3-anhydrosugar, ${ }^{30}$ de novo synthesis, ${ }^{31,32}$ oxidative activation with $\mathrm{I}_{2},{ }^{33}$ etc. However, their application in complex carbohydrate synthesis was usually case by case, hampered by narrow substrate scope, low efficiency, tedious steps, or harsh conditions. Even for the regular non-deoxy-glycosyl (e.g. glucosyl) donors adaptable to the well-developed 2-O-acyl participation strategy (Fig. 1b), ${ }^{34}$ the glycosylation could encounter the formation of 1,2orthoester by-products, and the later saponification for removing the acyl group would decompose those base-labile substrates. ${ }^{35}$ Hence, the development of new strategies for $\beta$-selective glycosylation is still in high demand, particularly for the efficient access to the biologically and synthetically important 2-deoxy- and 2-azido-2-deoxy- $\beta$-glycosides.
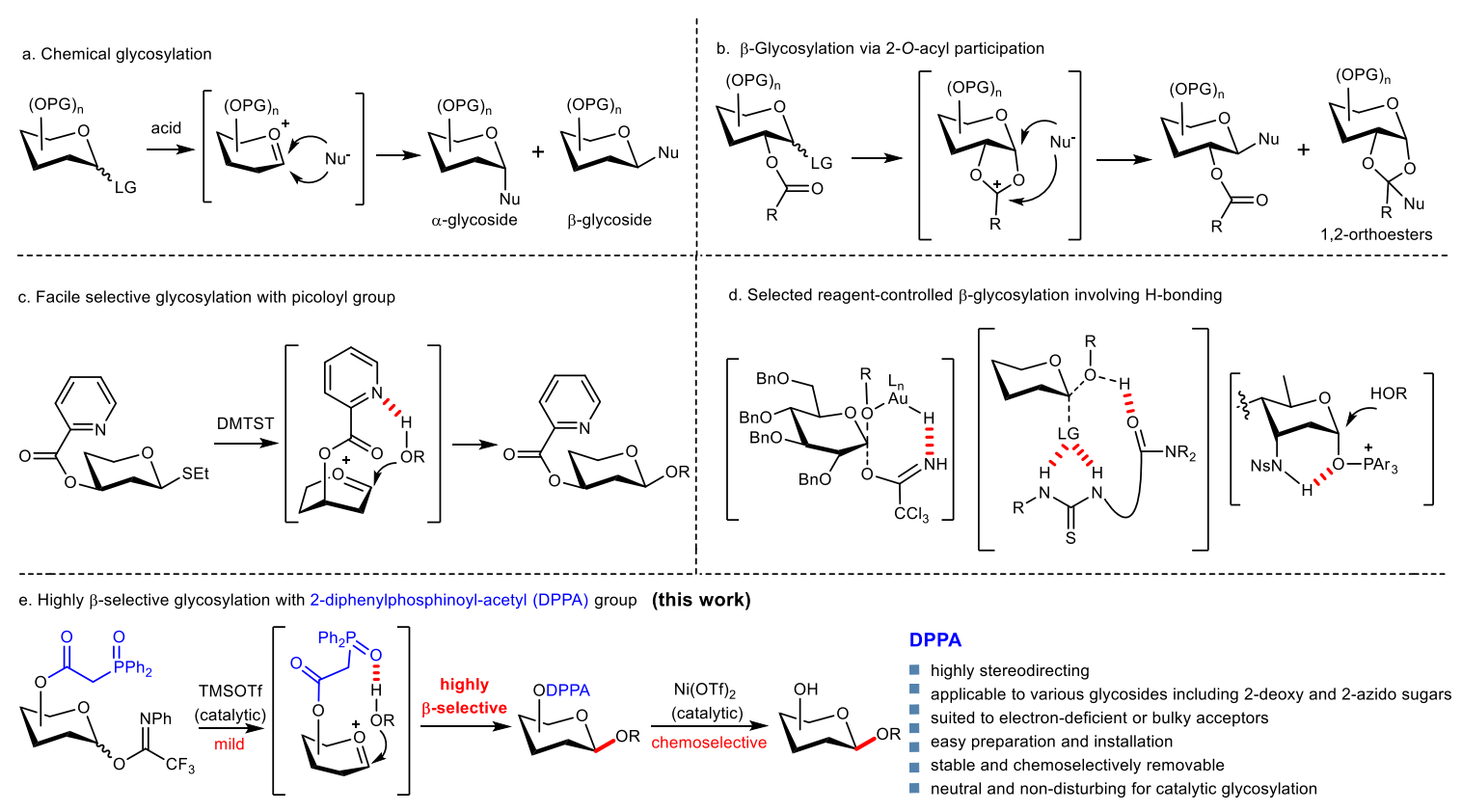

Fig. 1 Chemical glycosylation and selected strategies for $\beta$-glycosylation. PG: protecting group; LG: leaving group; 
DMTST: dimethyl(methylthio)sulfonium triflate; Nu: nucleophile; Ns: p-nitrobenzenesulfonyl.

Hydrogen bonding (H-bonding) could be utilized for the anomeric stereocontrol in glycosylation. ${ }^{5,36,37}$ In 2012, Demchenko and coworkers reported the use of picoloyl (Fig. 1c), a pyridine-containing group, to realize a facial selective glycosylation via the H-bond tethering between the pyridine moiety and the alcoholic acceptors. ${ }^{38}$ This process used thioglycosides as donors and a stoichiometric amount of dimethyl(methylthio)sulfonium triflate (DMTST) as promoter. Although they observed decreased stereoselectivity when electron-deficient or bulky acceptors were glycosylated, ${ }^{39}$ this elegant strategy has been successfully applied to prepare various glycosides including $\alpha$-sialic glycosides. ${ }^{40-48}$ Another $\mathrm{N}$-heterocyclic 2-quinolinecarbonyl group was employed by Yang and coworkers as H-bond-acceptor to effectively construct $\beta$-arabinofuranosides and $\alpha / \beta$-Kdo glycosides ${ }^{49,50} \mathrm{H}$-bonding also played an important role in several reagentcontrolled strategies for $\beta$-glycosylation using $\alpha$-glycosyl donors or intermediates (Fig. 1d). ${ }^{51-55}$

Compared to the basic pyridine and derivatives that could potentially interfere with glycosylation promoters, phosphine oxides are generally considered to be more neutral but stronger H-bond-acceptors. ${ }^{56}$ We questioned whether phosphine oxides would lead to better stereoselectivity and yields in catalytic glycosylation, aiming for the efficient preparation of the labile 2-deoxy- $\beta$-glycosides requiring mild reaction conditions. ${ }^{48}$ Thus, we envisioned several phosphine oxides (e.g. Fig. 1e) coupled to sugars via ester linkages, given the mild and easy formation of ester bonds as well as their good tolerance toward various conditions. Moreover, it would be feasible to selectively remove these phosphine oxidecontaining esters by the mild metal chelation instead of the basic saponification. ${ }^{57}$ The $\beta$-oriented $6-\mathrm{OH}$ of sugars would be selected as the coupling site for quick access to glycosyl donors. Furthermore, late-stage elaboration of a deprotected 6-OH such as glycosylation, oxidation, and dehydroxylation could readily provide $(1 \rightarrow 6)$-linked oligosaccharides, uronic acids, and 6-deoxy sugars, which are widespread in drugs and bioactive molecules (e.g. heparin). The other hydroxyl groups would be protected by benzyl groups rather than acyl groups to exclude the neighboring or remote participation of acyl groups in glycosylation. ${ }^{48}$ Herein, we report the development of a highly stereodirecting and chemoselectively removable 2-diphenylphosphinoyl-acetyl (DPPA) group for $\beta$-glycosylation (Fig. 1e). TMSOTf-catalyzed glycosylation with DPPA-installed glycosyl $N$-phenyltrifluoroacetimidate (PTFAI) donors displayed excellent $\beta$-selectivity and generality for the 2-deoxy/2-deoxy-2-azido sugars, as well as the non-deoxy sugars (e.g. glucose, galactose, mannose) as a good compensation for the conventional 2-O-acyl participation strategy to avoid orthoester formation and ester saponification. ${ }^{34,35}$

\section{Results}




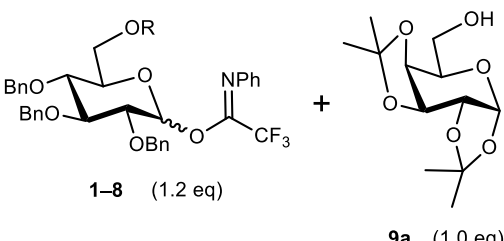

9 a $(1.0 \mathrm{eq})$

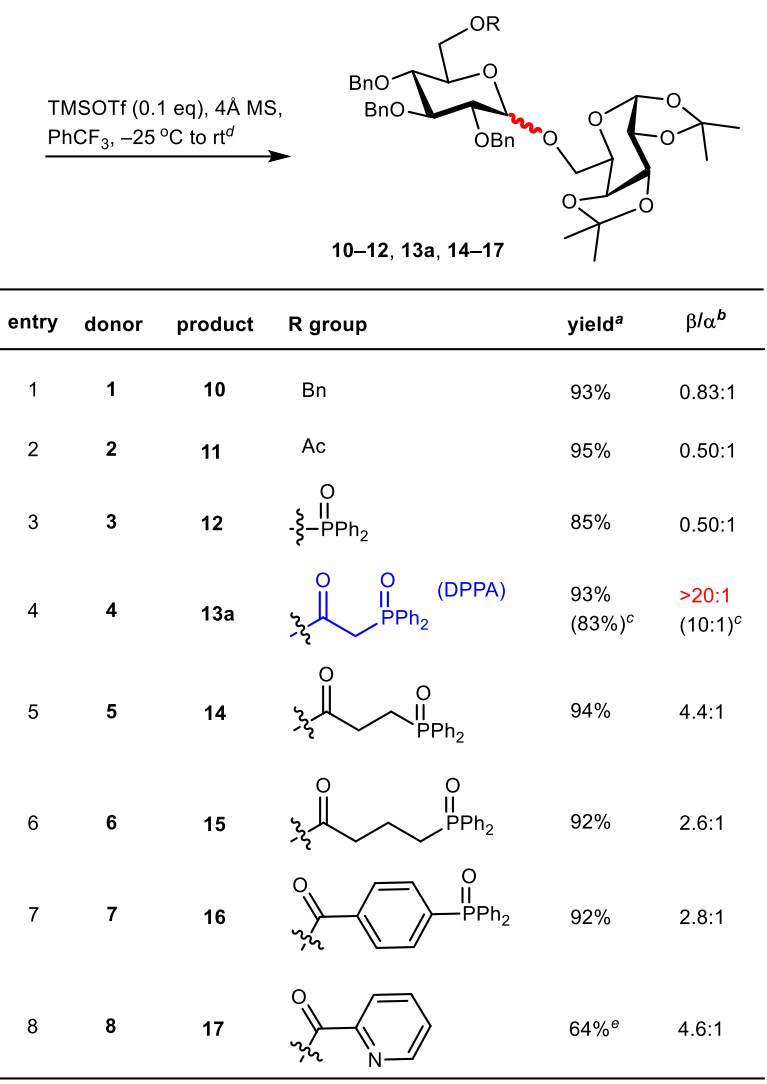

Fig. 2 Glycosylation of 9a with donors 1-8 varied at O6. ${ }^{a}$ Isolated by chromatography. ${ }^{b}$ Determined by ${ }^{1} \mathrm{H}$ NMR. ${ }^{c}$ The crude of donor 4 was directly used without the standard silica gel column purification. ${ }^{d}$ Warmed to rt from $-25^{\circ} \mathrm{C}$ in $1 \mathrm{~h}$, and stirred for another $1 \mathrm{~h}$ at $\mathrm{rt} .{ }^{e}$ Warmed to rt from $-25^{\circ} \mathrm{C}$ in $1 \mathrm{~h}$, and stirred overnight.

Screening for stereodirecting groups. Our studies commenced with the screening of glycosylation conditions using the easily accessible D-glucosyl donors (see SI for Table S1), giving the optimized conditions (1.2 eq of PTFAI donor, ${ }^{58-60}$ 0.1 eq of TMSOTf, $\mathrm{PhCF}_{3},{ }^{61,62} 4 \AA \mathrm{AS},-25{ }^{\circ} \mathrm{C}$ to rt). $\mathrm{PhCF}_{3}$, an alternative solvent to $\mathrm{CH}_{2} \mathrm{Cl}_{2}$, ${ }^{62}$ was found to provide the best $\beta$-selectivity with high yields. Next, a series of PTFAI donors (1-8, Fig. 2) bearing different substitutes at O6 were prepared (see SI for details), including five phosphine-oxide-containing acyl groups for H-bonding as well as benzyl/acetyl/picoloyl groups for control reactions. ${ }^{63-66}$ Their stereodirecting effects were then evaluated in the glycosylation of relatively hindered alcohol 9a. As expected, 6-OBn donor $\mathbf{1}$ was converted into disaccharide $\mathbf{1 0}$ with 
more $\alpha$-anomer (entry 1), while glycosylation with 6-OAc donor 2 showed even higher $\alpha$-selectivity $(\beta / \alpha=0.50: 1$, entry 2). ${ }^{67}$ Gratifyingly, we observed excellent $\beta$-selectivity when 6-ODPPA donor 4 was employed (entry 4 ), and the desired disaccharide 13a was obtained in a high $93 \%$ yield with the $\beta / \alpha$ ratio of more than $20: 1$. This $\beta$-selectivity could be attributed to an intermolecular aglycone delivery that was mediated by the H-bond tethering between the phosphine oxide on 4 and the 6-OH on 9a (Fig. 1e). ${ }^{38}$ The acetyl-derived linkage in DPPA group proved to be crucial for the high stereoselectivity, as further elongation with more flexible aliphatic (entries 5 and 6) or rigid aromatic linkage (entry 7) resulted in the decrease of $\beta / \alpha$ ratio. It is noteworthy that PTFAI donor $\mathbf{4}$ was prepared nearly as a single $\beta$-anomer using the standard protocol of $\mathrm{CF}_{3} \mathrm{C}(\mathrm{NPh}) \mathrm{Cl}$ and $\mathrm{K}_{2} \mathrm{CO}_{3}$ in acetone, implying that the high $\beta$-selectivity in the glycosylation with 4 was not caused by $S_{N} 2$ or $S_{N} 2$-like attack. To simplify the purification of donor $\mathbf{4}$, we collected its crude after a quick filtration and directly applied to glycosylation without the standard silica gel column purification. However, the resulting $\beta / \alpha$ ratio of disaccharide $\mathbf{1 3 a}$ decreased to $10: 1$ (entry 4), presumably due to the trace amount of the remaining $\mathrm{K}_{2} \mathrm{CO}_{3}$ that disturbed the pivotal $\mathrm{H}$-bonding. The effect of picoloyl group was also examined using 6-O-picoloyl PTFAI donor 8 in this catalytic glycosylation (entry 8 ), giving a modest $\beta$-selectivity ( $\beta / \alpha=4.6: 1$ ) with a dramatically decreased $64 \%$ yield, suggesting that the picoloyl group could disturb the TMSOTf-catalyzed glycosylation due to the basic pyridine moiety. 

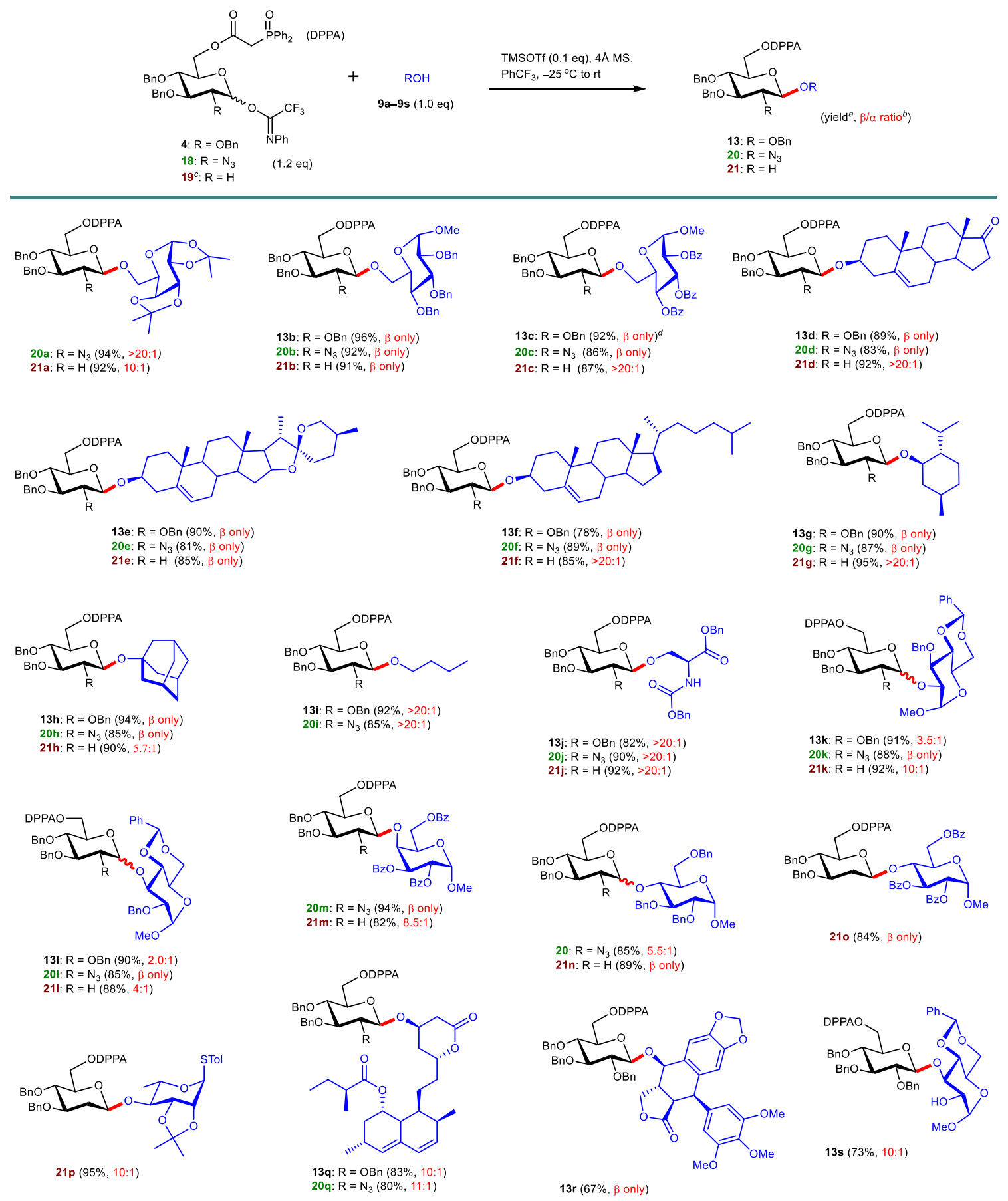

Fig. 3 Reaction scope with 6-ODPPA glycosyl donors 4, 18, and 19. ${ }^{a}$ Isolated by chromatography. ${ }^{b}$ Determined by ${ }^{1} \mathrm{H}$ NMR. ${ }^{c}$ Donor 19 was prepared and used without silica gel column purification. ${ }^{d}$ Scaled up.

Substrate scope. With 6-ODPPA confirmed as a promising $\beta$-directing group for glycosylation, the corresponding 2deoxy- and 2-azido-2-deoxy-glycosyl PTFAI donors 19 and 18 were prepared (see SI for details), respectively. Together with glucosyl donor 4, they were employed to glycosylate various alcohols as substrate scope examination (Fig. 3). The 
intrinsic high-reactivity of deoxy sugars made 2-deoxy imidate 19 not shelf-stable, and we used its crude for glycosylation without silica gel column purification. Nevertheless, all three donors generally led to excellent $\beta$-selectivity ( $\beta$ only or $\beta / \alpha>20: 1)$ and high yields in the glycosylation of reactive and moderately reactive alcohols $\mathbf{9 a}-\mathbf{9 j}$, including electronrich/electron-deficient glycosides 9b/9c, sterols 9d-9f, menthol 9g, adamantanol 9h, butanol 9i and serine 9j. The $\beta$ specific glycosylation of sapogenin aglycone 9d-9f showed a practical synthetic route to those $\beta$-linked 3-O-glycosyl saponins possessing diverse bioactivities (e.g. ginsenosides, polyphyllins, dioscin). ${ }^{68}$ It is particularly noteworthy that complete $\beta$-selectivity could be obtained when electron-deficient acceptor 9c was glycosylated, compared to the previously reported $\beta / \alpha$ ratio of 2.9:1 using the 6-O-picoloyl thioglycoside counterpart of donor $4 .{ }^{39}$ Additionally, this reaction could be readily scaled up, as demonstrated by the preparation of disaccharide 13c ( $\beta$ only, $0.94 \mathrm{~g}, 92 \%$ ). 2Deoxy donor 19 was found to be slightly less $\beta$-directing than $\mathbf{1 8}$ and $\mathbf{4}$ presumably owing to the lack of silica gel purification, but it still led to excellent $\beta$-selectivity in most cases, except in the preparation of 21a $(\beta / \alpha=10: 1)$ and $21 \mathbf{h}$ $(\beta / \alpha=5.7: 1)$ from structurally rigid alcohols $9 \mathbf{a}$ and $\mathbf{9 h}$. Further application to synthesize more complex and sophisticated glycoconjugates was successfully demonstrated in the conversion of antitumor podophyllotoxin (9r) and cholesterollowering drug lovastatin (9q) into glycosides 13r, 13q, and 20q with high $\beta$-selectivity and yields.

It has been well recognized that acceptors with poor reactivity, such as $9 \mathbf{k}-\mathbf{9 p}$, tend to give poor $\beta$-selectivity in glycosylation as a consequence of the steric hindrance or electron-withdrawing groups. ${ }^{38,39,49,51,69,70}$ Thus, we examined their performance in this DPPA-directed $\beta$-glycosylation (Fig. 3). As expected, the $\beta$-selectivity dropped to a modest range when benzylidene-tethered glucosides $9 \mathbf{k}$ and 91 were glycosylated with glucosyl donor $\mathbf{4}$, and 91 bearing the less reactive $3-\mathrm{OH}$ resulted in a lower $\beta / \alpha$ ratio $(2.0: 1$ for $\mathbf{1 3 l})$ than that of $2-\mathrm{OH}$ 9k $(3.5: 1$ for $\mathbf{1 3 k}) .{ }^{51}$ However, an unexpected regio- and stereo-selectivity was observed when 2,3-diol 9s was glycosylated with 1.2 eq of donor 4. Instead of the anticipative $(1 \rightarrow 2)$-linked disaccharide as the major product, we only obtained $(1 \rightarrow 3)$-linked disaccharide $13 \mathrm{~s}$ in $73 \%$ yield with a dramatically improved $\beta / \alpha$ ratio of 10:1, in comparison to the ratio of 2.0:1 for $\mathbf{1 3 1}$. The structure of the $\beta$ anomer of 13s was carefully characterized by 2D NMR spectra including COSY, HSQC, and HMBC, wherein the correlation between $\mathrm{H} 3$ and $\mathrm{C}^{\prime}$ ' as well as the $J_{\mathrm{H} 1}{ }^{\prime}, \mathrm{H} 2$ ' coupling constant of $7.8 \mathrm{~Hz}$ indicated the $\beta$ - $(1 \rightarrow 3)$-glycosidic linkage unambiguously. This interesting result might be related to a selective activation of 3-OH by $\mathrm{H}$-bonding, giving a promising way to realize the regio- and stereo-selective glycosylation of the less reactive 3-OH without shielding 2-OH.

On the other hand, 2-azido-2-deoxy donor 18 remained highly $\beta$-directing even in the glycosylation of the poor acceptors 9k and 9l, leading to the $\beta$-configured $20 \mathrm{k}$ and 201 in a stereospecific manner (Fig. 3). This could be attributed to the 
electron deficiency of azido group that could slow down the reaction and allow the sufficient formation of H-bond, implying that other electron-withdrawing groups such as acetyl and benzoyl on donors could also benefit the stereoselectivity. ${ }^{20}$ Moreover, it was particularly interesting to obtain $\mathbf{2 0 m}$ as a single $\beta$-anomer in the glycosylation of 4-OH galactoside 9m, one of the most electron-deficient and sterically hindered glycosyl acceptors. This complete $\beta$ selectivity could be a consequence of the enhancement of H-bonding due to the multiple electron-withdrawing benzoyl groups that made $\mathbf{9 m}$ a better H-bond-donor. ${ }^{71}$ Meanwhile, the enhanced H-bonding could also make the H-bonded $\mathbf{9 m}$ more nucleophilic to compete with the free $\mathbf{9 m}$ in glycosylation. This hypothesis was supported by the decreased $\beta / \alpha$ ratio of 5.1:1 when another bulky 4-OH glucoside $\mathbf{9 n}$ was glycosylated with donor $\mathbf{1 8}$, although $\mathbf{9 n}$ is usually considered to be more reactive than $\mathbf{9 m}$ and supposed to give better stereoselectivity because of its equatorial orientation of $4-\mathrm{OH}$ and multiple electron-donating benzyl groups.

In terms of the more challenging glycosylation of these poor acceptors with highly reactive 2-deoxy donor 19, it was gratifying to find that the 6-ODPPA group still led to excellent or good $\beta$-selectivity, and the corresponding disaccharides 21k-21p were afforded in high yields (Fig. 3). Notably, complete $\beta$-selectivity was obtained in the glycosylation of bulky acceptor 9n or 90 bearing glucosyl 4-OH, no matter electron-rich or electron-deficient. This could be attributed to the high flexibility of sugar ring that could quickly change the conformation to benefit the intermolecular H-bonding. Flexible galactoside $9 \mathrm{~m}$ bearing the even more bulky 4-OH could also be successfully converted into disaccharide $21 \mathrm{~m}$ with a satisfactory $\beta / \alpha$ ratio of $8.5: 1$. Replacement of $\mathbf{9 m}$ with more reactive acceptors $9 \mathbf{k}, \mathbf{9 1}$, and $\mathbf{9 p}$ did not significantly improve the $\beta$-selectivity presumably due to their rigid skeleton restrained by benzylidene or isopropylidene, but still giving disaccharides $\mathbf{2 1 k}, \mathbf{2 1 1}$, and $\mathbf{2 1 p}$ in high yields. This detrimental effect of rigidity on $\beta$-selectivity was also consistent with the decreased $\beta / \alpha$ ratio obtained in the preparation of $\mathbf{2 1 a}$ and $\mathbf{2 1 h}$ above.
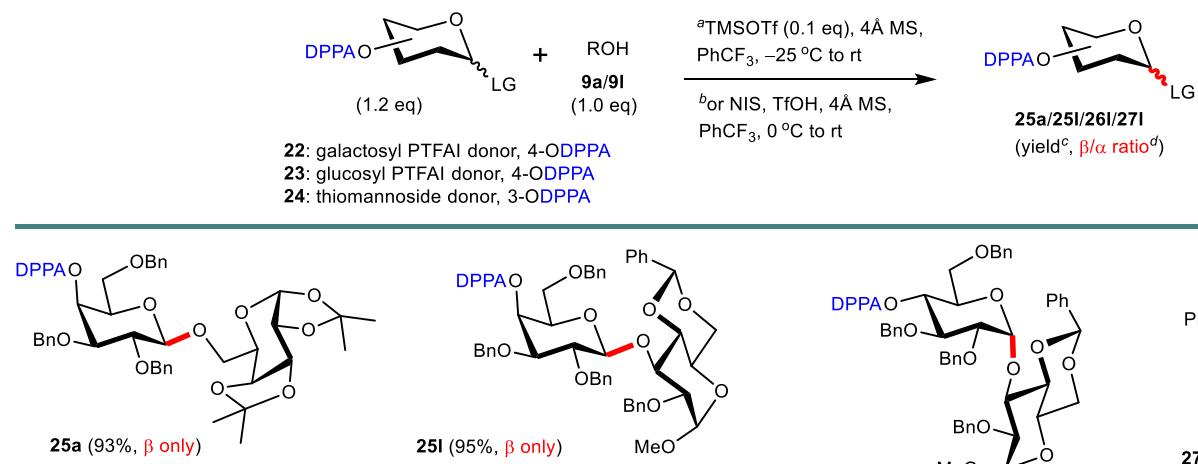

(yield $^{c}, \beta / \alpha$ ratio $^{d}$ )

syl PTFAI donor, 4-ODPPA
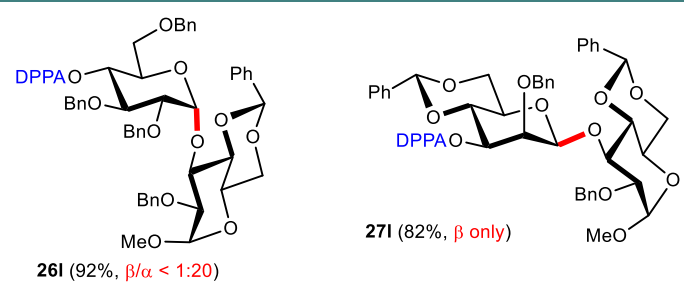

Fig. 4 Stereoselective glycosylation with galactosyl, glucosyl, and mannosyl donors 22-24 bearing 3- or 4-ODPPA 
group. ${ }^{a}$ For PTFAI donor 22 and 23. ${ }^{b}$ For thiomannoside donor 24. ${ }^{c}$ Isolated by chromatography. ${ }^{d}$ Determined by ${ }^{1} \mathrm{H}$ NMR.

To further understand the stereodirecting effect of DPPA group installed at O3 and O4 rather than O6, we prepared 4ODPPA galactosyl donor 22, 4-ODPPA glucosyl donor 23, and 3-ODPPA mannosyl donor 24 (see SI for details) to investigate their performance, particularly in the challenging $\alpha$-glucosylation and $\beta$-mannosylation. As depicted in Fig. 4, galactosyl PTFAI donor 22 was first condensed with alcohol 9a under the standard conditions (0.1 eq of TMSOTf, $\mathrm{PhCF}_{3}, 4 \AA \mathrm{MS},-25^{\circ} \mathrm{C}$ to rt), and disaccharide $\mathbf{2 5 a}$ was afforded in $93 \%$ yield with complete $\beta$-selectivity. For acceptor 91 that led to the poorest $\beta$-selectivity in the aforementioned studies (e.g. 13I and 201), the glycosylation with donor 22 still displayed complete $\beta$-selectivity and gave disaccharide $\mathbf{2 5 1}$ in $95 \%$ yield. On the contrary, a high $\alpha$-selectivity $(\alpha / \beta>20: 1)$ was observed when 91 was glycosylated with glucosyl donor $\mathbf{2 3}$ bearing the $\alpha$-oriented 4-ODPPA group, and the desired disaccharide $\mathbf{2 6 1}$ was obtained in $92 \%$ yield. Even the more challenging $\beta$-mannosylation of 91 with 3-ODPPA thiomannoside $\mathbf{2 4}$ was found to provide the desired $\mathbf{2 7 1}$ with complete $\beta$-selectivity, in contrast to the previously reported $\beta / \alpha$ ratio of 1.7:1 obtained with 91 and the 3-OBn counterpart of donor $\mathbf{2 4}$ under the similar conditions of NIS and TfOH. ${ }^{72}$ These results suggested that DPPA group installed at $\mathrm{O} 3$ and $\mathrm{O} 4$ was even more stereodirecting in glycosylation.

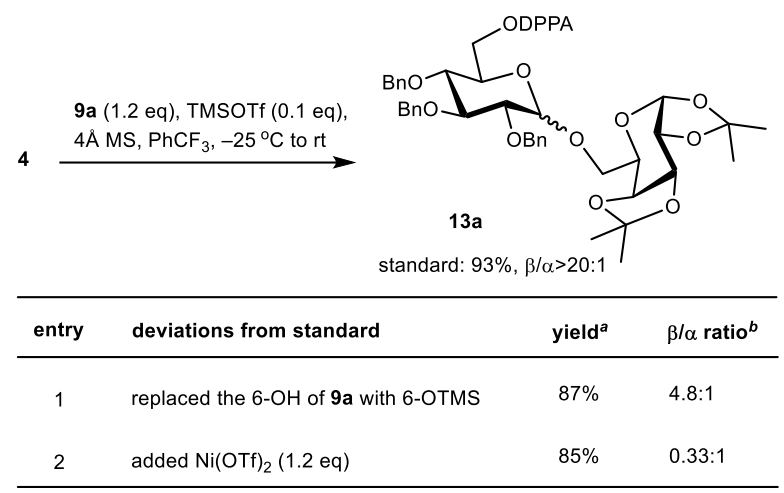

Fig. 5 Control reactions to verify the H-bonding. ${ }^{a}$ Isolated by chromatography. ${ }^{b}$ Determined by ${ }^{1} \mathrm{H}$ NMR.

H-bonding verification. The pivotal role of H-bonding in this stereoselective glycosylation was further verified with two more control reactions (Fig. 5), wherein the H-bonding would be interrupted by blocking either H-bond-donor or acceptor. Thus, the 6-OH of 9a was shielded by TMS and then applied to the condensation with donor $\mathbf{4}$ under the standard conditions (entry 1), and disaccharide 13a was obtained with the expected decreased $\beta / \alpha$ ratio of $4.8: 1$ compared to the original ratio of more than 20:1. In fact, the remaining $\beta$-selectivity could be caused by the partial TMS- 
deprotection during glycosylation, releasing the free 6-OH for H-bonding. ${ }^{38}$ The other control reaction was conducted with $9 \mathrm{a}$ and 4 but in the presence of 1.2 eq of $\mathrm{Ni}(\mathrm{OTf})_{2}$ that was envisaged to coordinate the phosphine oxide and block the H-bond-acceptor. Indeed, a reversed stereoselectivity was observed with the $\beta / \alpha$ ratio of $0.33: 1$.

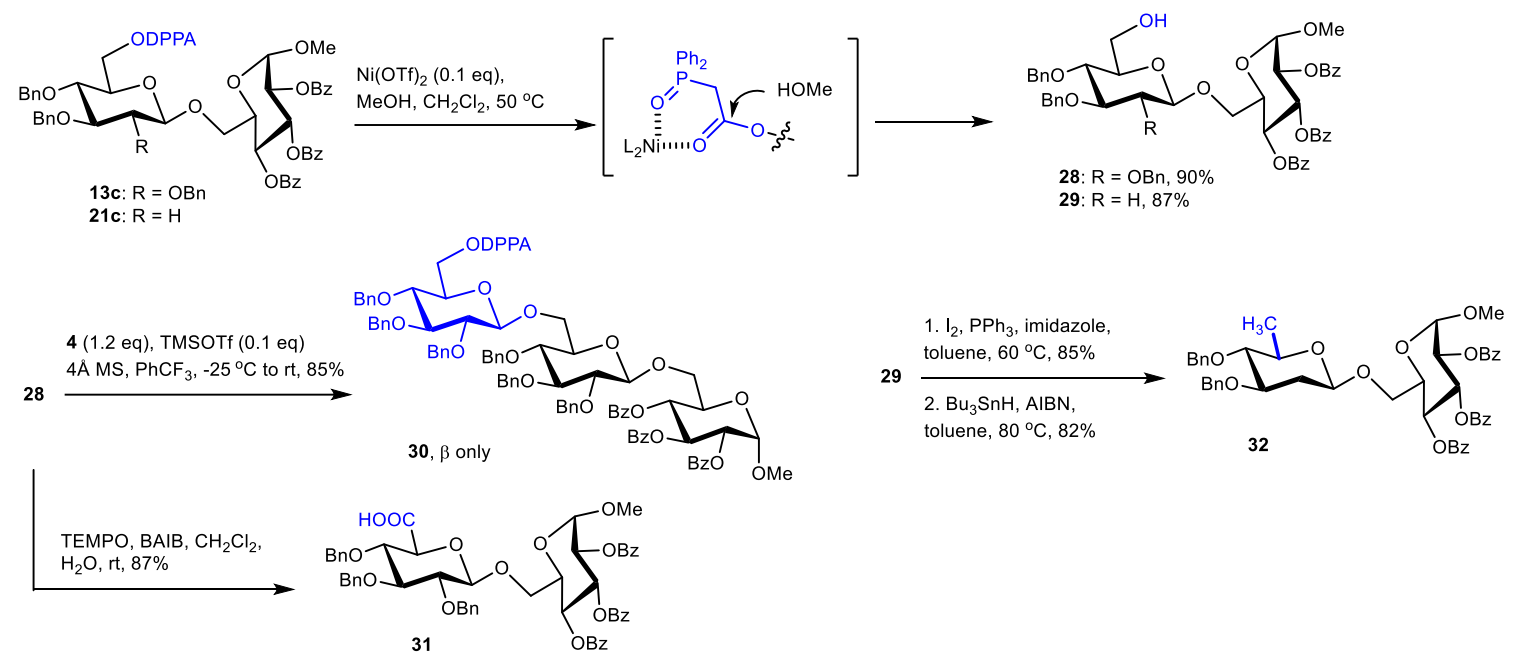

Fig. 6 Selective removal of DPPA group and further conversion to oligosaccharide, uronic acid, and 2,6-dideoxy sugar.

Chemoselective removal and further derivatization. Although the DPPA group could be easily removed under the regular saponification conditions such as $\mathrm{MeONa}$ in $\mathrm{MeOH}$, a chemoselective and mild removal without affecting other esters is highly desirable for those base-labile target products. Moreover, derivatization of the resulting alcohol could realize a convenient preparation of many biologically important molecules, such as $(1 \rightarrow 6)$-linked oligosaccharides, uronic acids, and 2,6-dideoxy- $\beta$-glycosides. Thus, we conducted a screening (see SI for Table S2) and found that a catalytic amount of $\mathrm{Ni}(\mathrm{OTf})_{2}$ could sufficiently remove the DPPA group in a mixture of $\mathrm{MeOH}$ and $\mathrm{CH}_{2} \mathrm{Cl}_{2}$ at $50{ }^{\circ} \mathrm{C}(\mathrm{Fig}$. 6). This chemoselective deprotection could be attributed to the putative chelation of $\mathrm{Ni}$ with the phosphine oxide and carbonyl group, making the carbonyl group electron-deficient and ready for the attack of methanol. ${ }^{57,73}$ Accordingly, disaccharides 13c and 21c bearing multiple base-labile benzoyl groups and acid-labile glycosidic linkages were smoothly converted into the corresponding alcohols $\mathbf{2 8}$ and $\mathbf{2 9}$ in around $90 \%$ yields. A further elongation was then carried out to glycosylate the resulting disaccharide 28 with another portion of donor $\mathbf{4}$, giving trisaccharide $\mathbf{3 0}$ in $85 \%$ yield with complete $\beta$-selectivity. Treatment of $\mathbf{2 8}$ with TEMPO and BAIB led to the desired uronic acid $\mathbf{3 1}$ in $87 \%$ yield. We also demonstrated an efficient conversion of 2-deoxy 29 into 2,6-dideoxy 32 via iodination and subsequent dehalogenation. 


\section{Discussion}

As a strong H-bond-acceptor, phosphine oxides were found neutral and non-disturbing for the catalytic glycosylation.

We have developed 2-diphenylphosphinoyl-acetyl (DPPA) group as a remote stereodirecting group for TMSOTfcatalyzed glycosylation via the H-bonding between the phosphine oxide and alcoholic acceptors. This DPPA group could be readily prepared and introduced to the $\mathrm{O} 6$ of glycosyl donors, directing excellent $\beta$-selectivity towards a wide range of acceptors including those with high steric hindrance and electron-deficiency. It is particularly practical for the stereoselective construction of the biologically and synthetically important 2-deoxy- and 2-azido-2-deoxy- $\beta$-glycosides, which represent one of the most difficult tasks in carbohydrate chemistry. DPPA group installed at O3 or O4 was also found highly stereodirecting in the challenging $\beta$-mannosylation and $\alpha$-glucosylation. As a temporary protecting group, the DPPA group could be chemoselectively removed with a catalytic amount of $\mathrm{Ni}(\mathrm{OTf})_{2}$ without affecting other acidor base-labile groups. Further elaboration of the resulting $6-\mathrm{OH}$ provides easy access to $(1 \rightarrow 6)$-linked oligosaccharides, uronic acids and 2,6-dideoxy- $\beta$-glycosides, which are important types of glycosides with diverse biological activities. We believe that this work would provide an alternative and inspiring strategy for the more concise and efficient synthesis of bioactive carbohydrate molecules.

\section{Acknowledgments}

This work was supported by the National Natural Science Foundation of China (21977115 and 21672248). We thank Professor Zhiyu Li and Professor Jianyi Wang for the helpful discussion of the manuscript.

\section{Author contributions}

W.L. designed the research and wrote the manuscript. X.L., Y.L., Q.S., G.L. and Y.S. conducted the synthetic work. H.S. contributed computational assistant. P.X., W.X., H.S. and B.Y. revised the manuscript and fully engaged in discussion.

\section{References}

1. Varki, A. Biological roles of glycans. Glycobiology 27, 3-49 (2017).

2. Dwek, R.A. Glycobiology: Toward understanding the function of sugars. Chem. Rev. 96, 683-720 (1996).

3. Zhu, X. \& Schmidt, R.R. New principles for glycoside-bond formation. Angew. Chem. Int. Ed. 48, 1900-1934 (2009).

4. Crich, D. Mechanism of a chemical glycosylation reaction. Acc. Chem. Res. 43, 1144-1153 (2010).

5. Leng, W.-L., Yao, H., He, J.-X. \& Liu, X.-W. Venturing beyond donor-controlled glycosylation: new perspectives toward anomeric selectivity. Acc. Chem. Res. 51, 628-639 (2018).

6. Nielsen, M.M. \& Pedersen, C.M. Catalytic glycosylations in oligosaccharide synthesis. Chem. Rev. 118, 82858358 (2018).

7. Guberman, M. \& Seeberger, P.H. Automated Glycan Assembly: A Perspective. J. Am. Chem. Soc. 141, 55815592 (2019).

8. Bennett, C.S. \& Galan, M.C. Methods for 2-deoxyglycoside synthesis. Chem. Rev. 118, 7931-7985 (2018). 
9. Zeng, J., Xu, Y., Wang, H., Meng, L. \& Wan, Q. Recent progress on the synthesis of 2-deoxy glycosides. Sci. China Chem. 60, 1162-1179 (2017).

10. Hou, D. \& Lowary, T.L. Recent advances in the synthesis of 2-deoxy-glycosides. Carbohydr. Res. 344, 19111940 (2009).

11. Marzabadi, C.H. \& Franck, R.W. The synthesis of 2-deoxyglycosides: 1988-1999. Tetrahedron 56, 8385-8417 (2000).

12. Borovika, A. \& Nagorny, P. Recent advances in the synthesis of natural 2-deoxy- $\beta$-glycosides. J. Carbohydr. Chem. 31, 255-283 (2012).

13. Li, Z., et al. Progress in the chemical synthesis of 2-deoxy-glycosides. Chin. J. Org. Chem. 32, 1812-1826 (2012).

14. Bongat, A.F.G. \& Demchenko, A.V. Recent trends in the synthesis of $O$-glycosides of 2-amino-2-deoxysugars. Carbohydr. Res. 342, 374-406 (2007).

15. Elshahawi, S.I., Shaaban, K.A., Kharel, M.K. \& Thorson, J.S. A comprehensive review of glycosylated bacterial natural products. Chem. Soc. Rev. 44, 7591-7697 (2015).

16. de Lederkremer, R.M. \& Marino, C. Deoxy sugars: occurrence and synthesis. Adv. Carbohydr. Chem. Biochem. 61, 143-216 (2007).

17. Beale, T.M., Moon, P.J. \& Taylor, M.S. Organoboron-catalyzed regio- and stereoselective formation of $\beta$-2deoxyglycosidic linkages. Org. Lett. 16, 3604-3607 (2014).

18. Issa, J.P. \& Bennett, C.S. A reagent-controlled $S_{\mathrm{N}} 2$-glycosylation for the direct synthesis of $\beta$-linked 2-deoxysugars. J. Am. Chem. Soc. 136, 5740-5744 (2014).

19. Plante, O.J., Palmacci, E.R. \& Seeberger, P.H. Formation of $\beta$-glucosamine and $\beta$-mannose linkages using glycosyl phosphates. Org. Lett. 2, 3841-3843 (2000).

20. van der Vorm, S., Overkleeft, H.S., van der Marel, G.A. \& Codée, J.D.C. Stereoselectivity of conformationally restricted glucosazide donors. J. Org. Chem. 82, 4793-4811 (2017).

21. Ma, Y., Li, Z., Shi, H., Zhang, J. \& Yu, B. Assembly of digitoxin by gold(I)-catalyzed glycosidation of glycosyl o-alkynylbenzoates. J. Org. Chem. 76, 9748-9756 (2011).

22 .

Baryal, K.N., Adhikari, S. \& Zhu, J. Catalytic stereoselective synthesis of $\beta$-digitoxosides: direct synthesis of digitoxin and C1'-epi-digitoxin. J. Org. Chem. 78, 12469-12476 (2013).

23. McDonald, F.E., Reddy, K.S. \& Díaz, Y. Stereoselective glycosylations of a family of 6-deoxy-1,2-glycals generated by catalytic alkynol cycloisomerization. J. Am. Chem. Soc. 122, 4304-4309 (2000).

24. Liu, C., Ma, Y., Pei, C., Li, W. \& Yu, B. A glycal approach to the synthesis of pregnane glycoside P57. Chin. J. Chem. 36, 1007-1010 (2018).

25. Roush, W.R. \& Bennett, C.E. A highly stereoselective synthesis of 2-deoxy- $\beta$-glycosides using 2-deoxy-2iodo-glucopyranosyl acetate donors. J. Am. Chem. Soc. 121, 3541-3542 (1999).

26. Yang, D.-M., Chen, Y., Sweeney, R.P., Lowary, T.L. \& Liang, X.-Y. Stereocontrolled synthesis of 2-deoxygalactopyranosides via isopropylidene-protected 6-O-silylated donors. Org. Lett. 20, 2287-2290 (2018).

27. Baryal, K.N., Zhu, D., Li, X. \& Zhu, J. Umpolung reactivity in the stereoselective synthesis of S-linked 2deoxyglycosides. Angew. Chem. Int. Ed. 52, 8012-8016 (2013).

28. Hoang, K.M., Lees, N.R. \& Herzon, S.B. Programmable synthesis of 2-deoxyglycosides. J. Am. Chem. Soc. 141, 8098-8103 (2019).

29. Zhu, D., Baryal, K.N., Adhikari, S. \& Zhu, J. Direct synthesis of 2-deoxy- $\beta$-glycosides via anomeric $O$ alkylation with secondary electrophiles. J. Am. Chem. Soc. 136, 3172-3175 (2014).

30. Hou, D. \& Lowary, T.L. 2,3-Anhydrosugars in glycoside bond synthesis. Application to 2,6dideoxypyranosides. J. Org. Chem. 74, 2278-2289 (2009).

31. Babu, R.S., Zhou, M. \& O'Doherty, G.A. De novo synthesis of oligosaccharides using a palladium-catalyzed glycosylation reaction. J. Am. Chem. Soc. 126, 3428-3429 (2004).

32. Seo, K. \& Rhee, Y.H. Ruthenium-catalyzed regioselective olefin migration of dihydropyran acetals: A de novo strategy toward $\beta$-2,6-dideoxypyranoglycosides. Org. Lett. 22, 2178-2181 (2020).

33. Tanaka, H., Yoshizawa, A. \& Takahashi, T. Direct and stereoselective synthesis of $\beta$-linked 2,6deoxyoligosaccharides. Angew. Chem. Int. Ed. 46, 2505-2507 (2007).

34. Hettikankanamalage, A.A., Lassfolk, R., Ekholm, F.S., Leino, R. \& Crich, D. Mechanisms of stereodirecting participation and ester migration from near and far in glycosylation and related reactions. Chem. Rev. 120, 7104-7151 (2020).

35. Christensen, H.M., Oscarson, S. \& Jensen, H.H. Common side reactions of the glycosyl donor in chemical glycosylation. Carbohydr. Res. 408, 51-95 (2015).

36. Whitfield, D.M. \& Guo, J. Proton transfer and hydrogen bonding in glycosylation reactions. J. Carbohydr. Chem. 36, 59-99 (2017).

37. Fang, T., Gu, Y., Huang, W. \& Boons, G.-J. Mechanism of glycosylation of anomeric sulfonium ions. J. Am. Chem. Soc. 138, 3002-3011 (2016).

38. Yasomanee, J.P. \& Demchenko, A.V. Effect of remote picolinyl and picoloyl substituents on the stereoselectivity of chemical glycosylation. J. Am. Chem. Soc. 134, 20097-20102 (2012).

39. Mannino, M.P., Yasomanee, J.P. \& Demchenko, A.V. Investigation of the H-bond-mediated aglycone delivery 
reaction in application to the synthesis of b-glucosides. Carbohydr. Res. 470, 1-7 (2018).

40. Yasomanee, J.P. \& Demchenko, A.V. Hydrogen bond mediated aglycone delivery: synthesis of linear and branched a-glucans. Angew. Chem. Int. Ed. 53, 10453-10456 (2014).

41. Pistorio, S.G., Yasomanee, J.P. \& Demchenko, A.V. Hydrogen-bond-mediated aglycone delivery: focus on bmannosylation. Org. Lett. 16, 716-719 (2014).

42. Escopy, S., Geringer, S.A. \& De Meo, C. Combined effect of the picoloyl protecting group and triflic acid in sialylation. Org. Lett. 19, 2638-2641 (2017).

43. Wu, Y.-F. \& Tsai, Y.-F. Assistance of the C-7,8-picoloyl moiety for directing the glycosyl acceptors into the a-orientation for the glycosylation of sialyl donors. Org. Lett. 19, 4171-4174 (2017).

44. Chen, J., et al. 1-Picolinyl-5-azido thiosialosides: Versatile donors for the stereoselective construction of sialyl linkages. Angew. Chem. Int. Ed. 58, 17000-17008 (2019).

45. Liu, D.-M., Wang, H.-L., Lei, J.-C., Zhou, X.-Y. \& Yang, J.-S. A Highly a-stereoselective sialylation method using 4-O-4-nitropicoloyl thiosialoside donor. Eur. J. Org. Chem., 575-585 (2020).

46. Wang, P., et al. Hydrogen-bond-mediated aglycone delivery: synthesis of b-D-fructofuranosides. Org. Lett. 22, 2967-2971 (2020).

47. Gucchait, A., Kundu, M., Manna, T., Shit, P. \& Misra, A.K. Influence of functional groups towards the $\beta$ selective glycosylation of 2-azido-2-deoxy glycosyl thioglycosides. Eur. J. Org. Chem., 3398-3409 (2020).

48. Ruei, J.-H., Venukumar, P., Ingle, A.B. \& Mong, K.-K.T. C6 picoloyl protection: a remote stereodirecting group for 2-deoxy- $\beta$-glycoside formation. Chem. Commun. 51, $5394-5397$ (2015).

49. Liu, Q.-W., Bin, H.-C. \& Yang, J.-S. b-Arabinofuranosylation using 5-O-(2-quinolinecarbonyl) substituted ethyl thioglycoside donors. Org. Lett. 15, 3974-3977 (2013).

50. Huang, W., et al. Stereodirecting effect of C5-carboxylate substituents on the glycosylation stereochemistry of 3-deoxy-D-manno-oct-2-ulosonic acid (Kdo) thioglycoside donors: stereoselective synthesis of a- and b-Kdo glycosides. J. Am. Chem. Soc. 140, 3574-3582 (2018).

51. Peng, P. \& Schmidt, R.R. An alternative reaction course in $O$-glycosidation with $O$-glycosyl trichloroacetimidates as glycosyl donors and lewis acidic metal salts as catalyst: acid-base catalysis with gold chloride-glycosyl acceptor adducts. J. Am. Chem. Soc. 137, 12653-12659 (2015).

52. Peng, P. \& Schmidt, R.R. Acid-base catalysis in glycosidations: a nature derived alternative to the generally employed methodology. Acc. Chem. Res. 50, 1171-1183 (2017).

53. Park, Y., et al. Macrocyclic bis-thioureas catalyze stereospecific glycosylation reactions. Science 355, $162-166$ (2017).

54. Li, Q., Levi, S.M. \& Jacobsen, E.N. Highly selective b-mannosylations and b-rhamnosylations catalyzed by bis-thiourea. J. Am. Chem. Soc. 142, 11865-11872 (2020).

55. Zeng, J., et al. Hydrogen-bonding-assisted exogenous nucleophilic reagent effect for b-selective glycosylation of rare 3-amino sugars. J. Am. Chem. Soc. 141, 8509-8515 (2019).

56. Graton, J., Le Questel, J.-Y., Maxwell, P. \& Popelier, P. Hydrogen-bond accepting properties of new heteroaromatic ring chemical motifs: a theoretical study. J. Chem. Inf. Model. 56, 322-334 (2016).

57. Kumar, N., Kachroo, P.L. \& Kant, R. Studies on complexes of metal(II) carboxylates with donor molecules. IV. Complexes of nickel(II) chloroacetates with pyridine $N$-oxides and triphenylphosphine oxide. Bull. Chem. Soc. Jpn. 53, 1787-1788 (1980).

58. Yu, B. \& Tao, H. Glycosyl trifluoroacetimidates. Part 1: preparation and application as new glycosyl donors. Tetrahedron Lett. 42, 2405-2407 (2001).

59. Yu, B. \& Sun, J. Glycosylation with glycosyl $N$-phenyltrifluoroacetimidates (PTFAI) and a perspective of the future development of new glycosylation methods. Chem. Commun. 46, 4668-4679 (2010).

60. Li, W., et al. Synthesis of bradyrhizose oligosaccharides relevant to the bradyrhizobium O-antigen. Angew. Chem. Int. Ed. 56, 2092-2096 (2017).

61. Carrel, F.R., Geyer, K., Codée, J.D.C. \& Seeberger, P.H. Oligosaccharide synthesis in microreactors. Org. Lett. 9, 2285-2288 (2007).

62. Ogawa, A. \& Curran, D.P. Benzotrifluoride: a useful alternative solvent for organic reactions currently conducted in dichloromethane and related solvents. J. Org. Chem. 62, 450-451 (1997).

63. Tsvetkov, E.N., Bondarenko, N.A., Malakhova, I.G. \& Kabachnik, M.I. A simple synthesis and some synthetic applications of substituted phosphide and phosphinite anions. Synthesis, 198-208 (1986).

64. Bevan, J.G.M., Lourenço, E.C., Chaves-Ferreira, M., Rodrigues, J.A. \& Ventura, M.R. Immobilization of UDP-galactose on an amphiphilic resin. Eur. J. Org. Chem., 908-914 (2018).

65. Hsu, M.-Y., Liu, Y.-P., Lam, S., Lin, S.-C. \& Wang, C.-C. TMSBr-mediated solvent- and work-up-free synthesis of a-2-deoxyglycosides from glycals. Beilstein J. Org. Chem. 12, 1758-1764 (2016).

66. Narayanan, K.S. \& Berlin, K.D. Novel synthesis of w-(diphenylphosphinyl)alkylcarboxylic acids from triphenyl-w-carboxyalkylphosphonium salts. J. Org. Chem. 45, 2240-2243 (1980).

67. Li, J., Li, W. \& Yu, B. A divergent approach to the synthesis of simplexides and congeners via a late-stage olefin cross-metathesis reaction. Org. Biomol. Chem. 11, 4971-4974 (2013).

68. Li, W. \& Yu, B. Gold-catalyzed glycosylation in the synthesis of complex carbohydrate-containing natural products. Chem. Soc. Rev. 47, 7954-7984 (2018). 
69. van der Vorm, S., et al. Acceptor reactivity in glycosylation reactions. Chem. Soc. Rev. 48, 4688-4706 (2019).

70. van der Vorm, S., et al. Mapping the relationship between glycosyl acceptor reactivity and glycosylation stereoselectivity. Angew. Chem. Int. Ed. 57, 8240-8244 (2018).

71. Le Mai Hoang, K. \& Liu, X.-W. The intriguing dual-directing effect of 2-cyanobenzyl ether for a highly stereospecific glycosylation reaction. Nat. Commun. 5, 5051 (2014).

72. Chang, C.-W., et al. Establishment of guidelines for the control of glycosylation reactions and intermediates by quantitative assessment of reactivity. Angew. Chem. Int. Ed. 58, 16775-16779 (2019).

73. Geringer, S.A., Mannino, M.P., Bandara, M.D. \& Demchenko, A.V. Picoloyl protecting group in synthesis: focus on a highly chemoselective catalytic removal. Org. Biomol. Chem. 18, 4863-4871 (2020). 